\title{
Some bounds on the distance-sum-connectivity matrix
}

\section{Gülistan Kaya Gök ${ }^{1 *}$}

\section{"Correspondence:}

gulistankayagok@hakkari.edu.tr

${ }^{1}$ Department of Mathematics

Education, Hakkari University,

Hakkari, Turkey

\section{Springer}

\begin{abstract}
The distance-sum-connectivity matrix of a graph $G$ is expressed by $\delta(i)$ and $\delta(j)$ such that $i, j \in V . \delta(i)$ and $\delta(j)$ are represented by a sum of the distance matrices for $i<v$ and $j<v$, respectively.

The purpose of this paper is to give new inequalities involving the eigenvalues, the graph energy, the graph incidence energy, and the matching energy. So, we have some results in terms of the edges, the vertices, and the degrees.
\end{abstract}

MSC: $05 C 22 ; 05 C 50$

Keywords: Distance-sum-connectivity matrix; Bounds

\section{Introduction}

Let $G$ be a simple, finite, connected graphs with the vertex set $V(G)$ and the edge set $E(G)$. By $d_{i}$ we denote the degree of a vertex. Throughout this paper, the vertex degrees are assumed to be ordered non-increasingly. The maximum and the minimum vertex degrees in a graph are denoted by $\Delta$ and $\delta$, respectively. If any vertices $i$ and $j$ are adjacent, then we use the notation $i \sim j$.

The distance-sum-connectivity matrix is defined by the displacement of the vertex degrees and the distance sum. This matrix is denoted by ${ }^{\delta} X$ and represented in [1-18] by

$$
{ }^{\delta} X= \begin{cases}(\delta(i) \delta(j))^{\frac{-1}{2}} & \text { if } i \sim j, \\ 0 & \text { otherwise. }\end{cases}
$$

The distance sum is $\delta(i)=\sum_{j=1}^{v}\left({ }^{v} D_{i j}\right)$ such that $\mathrm{D}$ is the distance matrix. The distancesum-connectivity matrix is an interesting matrix, and this paper deals with bounds of this matrix. We find some upper bounds and these bounds contain the edge numbers, the vertex numbers, and the eigenvalues. The eigenvalues of this matrix are $\lambda_{1}\left({ }^{\delta} X\right), \lambda_{2}\left({ }^{\delta} X\right), \ldots, \lambda_{n}\left({ }^{\delta} X\right)$ such that $\lambda_{1}\left({ }^{\delta} X\right) \geq \lambda_{2}\left({ }^{\delta} X\right) \geq \cdots \geq \lambda_{n}\left({ }^{\delta} X\right)$. We will accept $\lambda_{1}\left({ }^{\delta} X\right)$ as the spectral radius of the graph ${ }^{\delta} X(G)$, and we will take $\lambda_{1}\left({ }^{\delta} X\right)$ as $\lambda_{1}$ for convenience. Basic properties of $\lambda_{i}$ are $\sum_{i=1}^{n} \lambda_{i}=0, \sum_{i=1}^{n} \lambda_{i}^{2}=2 m$, and $\operatorname{det}\left({ }^{\delta} X\right)=\prod_{i=1}^{n} \lambda_{i}$. G is a regular graph with order $n$ if and only if $\lambda_{1} \geq \frac{2 m}{n}$ [3]. The energy of $\left({ }^{\delta} X\right)$ is described as $E\left({ }^{\delta} X\right)=\sum_{i=1}^{n}\left|\lambda_{i}(G)\right|$. Some properties about the graph energy may be found $[7,10]$. The incidence energy $I E$ of $G$ is introduced by Joojondeh et al. [13] as the sum of singular

(c) The Author(s) 2018. This article is distributed under the terms of the Creative Commons Attribution 4.0 International License (http://creativecommons.org/licenses/by/4.0/), which permits unrestricted use, distribution, and reproduction in any medium, provided you give appropriate credit to the original author(s) and the source, provide a link to the Creative Commons license, and indicate if changes were made. 
values of the incidence matrix of $G$. The incidence matrix of a graph $G$ is defined as

$$
I(G)= \begin{cases}1 & \text { if } v_{i} \text { is incident with } v_{j} \\ 0 & \text { otherwise }\end{cases}
$$

The singular values are $q_{1}\left({ }^{\delta} X\right), q_{2}\left({ }^{\delta} X\right), \ldots, q_{n}\left({ }^{\delta} X\right)$ such that $q_{1}\left({ }^{\delta} X\right) \geq q_{2}\left({ }^{\delta} X\right) \geq \cdots \geq$ $q_{n}\left({ }^{\delta} X\right)$. We use $q_{i}\left({ }^{\delta} X\right)$ as $q_{i}$ for brevity. The incidence energy of a graph is represented by $I E=I E(G)=\sum_{i=1}^{n} \sqrt{q_{i}(G)}$. See [8] and [9].

The number of $k$-matchings of a graph $G$ is denoted by $m(G, k)$. The matching polynomial of a graph is described by $\alpha(G)=\alpha(G, \lambda)=\sum_{k \geq 0}(-1)^{k} m(G, k) \lambda^{n-2 k}$ (see [6]).

The matching energy of a graph $G$ is defined as

$$
M E=M E(G)=\frac{2}{p i} \int_{o}^{\infty} \frac{1}{x^{2}} \ln \left[\sum_{k \geq 0} m(G, k) x^{2 k}\right] d x \quad \text { (see [11]). }
$$

The paper is planned as follows. In Sect. 2, we explain previous works. In the next section, we give a survey on upper bound for the first greatest eigenvalue $\lambda_{1}$ and the second greatest eigenvalue $\lambda_{2}$ using the edge number, the vertex number, and the degree. In Sect. 3.2, we focus on the upper bound for energy of ${ }^{\delta} X(G)$ and are concerned with the vertex number, the distance matrix, and the determinant of ${ }^{\delta} X$. In addition, we deal with some results for the incidence energy of ${ }^{\delta} X(G)$, and we find sharp inequalities of $I E\left({ }^{\delta} X(G)\right)$. In Sect. 3.3, we determine different results for the matching energy of a graph with some fixed parameters.

\section{Preliminaries}

In order to achieve our plan, we need the following lemmas and theorems.

Lemma 2.1 ([12]) Let $\lambda_{1}(A)$ be a spectral radius and $A=\left(a_{i j}\right)$ be an irreducible nonnegative matrix with $R_{i}(A)=\sum_{j=1}^{m} a_{i j}$. Then

$$
\left(\min R_{i}(A): 1 \leq i \leq n\right) \leq \lambda_{1}(A) \leq\left(\max R_{i}(A): 1 \leq i \leq n\right) .
$$

Lemma 2.2 ([4]) If $G$ is a simple, connected graph and $m_{i}$ is the average degree of the vertices adjacent to $v_{i} \in V$, then

$$
\lambda_{1}(G) \leq \max \left(\sqrt{m_{i} m_{j}}: 1 \leq i, j \leq n, v_{i}, v_{j} \in E\right) .
$$

Ozeki established Ozeki's inequality in [16]. This inequality holds some bounds for our graph energy. This inequality is as follows.

Theorem 2.3 (Ozeki's inequality) If $a_{i}, b_{i} \in R^{+}, 1 \leq i \leq n$, then

$$
\sum_{i=1}^{n} a_{i}^{2} \sum_{i=1}^{n} b_{i}^{2}-\left(\sum_{i=1}^{n} a_{i} b_{i}\right)^{2} \leq \frac{n^{2}}{4}\left(M_{1} M_{2}-m_{1} m_{2}\right)^{2},
$$

where $M_{1}=\max _{1 \leq i \leq n} a_{i}, M_{2}=\max _{1 \leq i \leq n} b_{i}, m_{1}=\min _{1 \leq i \leq n} a_{i}$, and $m_{2}=\max _{1 \leq i \leq n} b_{i}$. 
Polya-Szego found an interesting inequality in [17]. This inequality is set as follows.

Theorem 2.4 (Polya-Szego inequality) If $s_{i}, t_{i} \in R^{+}$for $1 \leq i \leq n$, then

$$
\sum_{i=1}^{n} s_{i}^{2} \sum_{i=1}^{n} t_{i}^{2} \leq \frac{1}{4}\left(\sqrt{\frac{K_{1} K_{2}}{k_{1} k_{2}}}+\sqrt{\frac{k_{1} k_{2}}{K_{1} K_{2}}}\right)^{2}\left(\sum_{i=1}^{n} s_{i} t_{i}\right)^{2}
$$

where $K_{1}=\max _{1 \leq i \leq n} s_{i}, K_{2}=\max _{1 \leq i \leq n} t_{i}, k_{1}=\min _{1 \leq i \leq n} s_{i}$, and $k_{2}=\max _{1 \leq i \leq n} t_{i}$.

Let $G$ be a simple graph and $X$ and $Y$ be any real symmetric matrices of $G$. Let us consider eigenvalues of these matrices. These eigenvalues hold in the following lemma.

Lemma 2.5 ([5]) Let $M$ and $N$ be two real symmetric matrices and $1 \leq \ell \leq n$, then

$$
\sum_{i=1}^{\ell} \lambda_{i}(M+N) \leq \sum_{i=1}^{\ell} \lambda_{i}(M)+\sum_{i=1}^{\ell} \lambda_{i}(N)
$$

Let $x_{1}, x_{2}, \ldots, x_{s}$ be positive real numbers for $1 \leq t \leq s . M_{t}$ is defined as follows:

$$
\begin{aligned}
& M_{1}=\frac{x_{1}+x_{2}+\cdots+x_{s}}{s}, \\
& M_{2}=\frac{x_{1} x_{2}+x_{1} x_{3}+\cdots+x_{1} x_{s}+x_{2} x_{3}+\cdots+x_{s-1} x_{s}}{\frac{1}{2} s(s-1)}, \\
& \cdots \\
& M_{s-1}=\frac{x_{1} x_{2}+\cdots+x_{s-1}+x_{1} x_{2}+\cdots+x_{s-2} x_{s}+\cdots+x_{2} x_{3}+\cdots+x_{s-1} x_{s}}{s}, \\
& M_{s}=x_{1} x_{2} \cdots x_{s} .
\end{aligned}
$$

Lemma 2.6 (Maclaurin's symmetric mean inequality [2]) Let $x_{1}, x_{2}, \ldots, x_{s}$ be real nonnegative numbers, then

$$
M_{1} \geq M_{2}^{\frac{1}{2}} \geq M_{3}^{\frac{1}{3}} \geq \cdots \geq M_{s}^{\frac{1}{s}}
$$

This equality holds if and only if $x_{1}=x_{2}=\cdots=x_{s}$.

Theorem 2.7 Let G be a simple graph. Let zeros of the matching polynomial of this graph be $\mu_{1}, \mu_{2}, \ldots, \mu_{n}$. Then

$$
\operatorname{ME}(G)=\sum_{i=1}^{n}\left|\mu_{i}\right|
$$

The zeros of the matching polynomial provide the equations $\sum_{i=1}^{n} \mu_{i}^{2}=2 m$ and $\sum_{i<j} \mu_{i} \mu_{j}=$ $-m$. 


\section{Main results}

\subsection{Upper bounds on eigenvalues}

A lot of bounds for the eigenvalues have been found. We now establish further bounds for $\lambda_{1}$ and $\lambda_{2}$ involving the $n, m$ and $d$. Firstly we give some known bounds about graph theory.

In the reference [14] a lower bound is given:

$$
E(G) \geq 2 \sqrt{m}
$$

if and only if $G$ consists of a complete bipartite graph $K_{x, y}$. In this note, $x y=m$.

Indeed, McClelland's famous bound is [15] $E(G) \leq \sqrt{2 m n}$.

We now will give an upper bound for the eigenvalues of ${ }^{\delta} X(G)$.

Theorem 3.1 If $G$ is a simple, connected graph and $D$ is the distance matrix of $G$, then

$$
\lambda_{1}(G) \leq \frac{1}{\sqrt[4]{D_{\text {in }} D_{j n}}} .
$$

Proof Let $X=\left(x_{1}, x_{2}, \ldots, x_{n}\right)^{T}$ be an eigenvector of $D(G)^{-1}\left({ }^{\delta} X(G)\right) D(G)$. Let one eigencomponent $x_{i}=1$ and the other eigencomponent $0<x_{k} \leq 1$ for every $k$. Let $x_{j}=$ $\max _{k}\left(x_{k}: v_{i} v_{k} \in E, i \sim k\right)$. We know $\left(D(G)^{-1}\left({ }^{\delta} X(G)\right) D(G)\right) X=\lambda_{1}(G) X$. If we take the $i$ th equation of this equation, we obtain

$$
\begin{aligned}
\lambda_{1}(G) x_{i} & =\sum_{k}(\delta(i) \delta(k))^{\frac{-1}{2}} x_{k} \\
& =\sum_{k}\left(\sum_{j=1}^{v}\left({ }^{v} D_{i j}\right) \sum_{t=1}^{v}\left({ }^{v} D_{k t}\right)\right)^{\frac{-1}{2}} x_{k} \\
& =\sum_{k}\left(\frac{1}{\sqrt{\sum_{j=1}^{v}\left({ }^{v} D_{i j}\right)}} \frac{1}{\sqrt{\sum_{t=1}^{v}\left({ }^{v} D_{k t}\right)}}\right) x_{k} .
\end{aligned}
$$

We can take each $D_{i j}$ 's as $D_{i n}$. So,

$$
\lambda_{1}(G) x_{i} \leq\left(\frac{1}{\sqrt{n D_{i n}}} \sum_{k}\left(\frac{1}{\sqrt{\left.\sum_{t=1}^{v}{ }^{(} D_{k t}\right)}}\right)\right) x_{k} .
$$

Using the Cauchy-Schwarz inequality,

$$
\begin{aligned}
\lambda_{1}(G) x_{i} & =\left(\frac{1}{\sqrt{n D_{i n}}}\right)\left(\frac{n}{\sqrt{n}}\right) x_{k} \\
& =\left(\frac{1}{\sqrt{D_{\text {in }}}}\right) x_{k} .
\end{aligned}
$$

From Lemmas 2.1 and 2.2, we have

$$
\lambda_{1}(G) \leq \sqrt{\frac{1}{\sqrt{D_{i n}}} \frac{1}{\sqrt{D_{j n}}}}
$$




$$
\leq \frac{1}{\sqrt[4]{D_{i n} D_{j n}}}
$$

Theorem 3.2 Let $G$ be a simple, connected graph with $m$ edges and $n$ vertices. Let $\lambda_{1}, \lambda_{2}, \ldots, \lambda_{n}$ be eigenvalues of the distance-sum-connectivity matrix ${ }^{\delta} X$ and $E(G)$ be an energy of ${ }^{\delta} X$, then

$$
\lambda_{2}(G) \leq \sqrt{\frac{2 m\left(\sqrt{2 m n}-d_{1}\right)}{n^{3} d_{1}}-\frac{4 m^{2}}{n^{6} d_{1}^{2}}+m},
$$

where $\lambda_{2}$ is the second greatest eigenvalue of ${ }^{\delta} X$.

Proof $\lambda_{2}$ is the second largest eigenvalue of ${ }^{\delta} X$. Firstly, we show that $\lambda_{1} \geq \frac{2 m}{n^{3} d_{1}}$. We know that $\left(D(G)^{-1}\left({ }^{\delta} X(G)\right) D(G)\right) X=\lambda_{1}(G) X$. So, $\lambda_{1}(G) x_{i}=\sum_{k}\left((\delta(i) \delta(j))^{\frac{-1}{2}} \frac{d_{k}}{d_{1}}\right) x_{k}$. Similar to Theorem 3.1, if we take the $i$ th equation of this equation, we obtain

$$
\begin{aligned}
\lambda_{1}(G) x_{i} & =\left(\sum_{k}\left(\sum_{m=1}^{v}\left({ }^{v} D_{i m}\right) \sum_{s=1}^{v}\left({ }^{v} D_{j s}\right)\right)^{\frac{-1}{2}} \frac{d_{k}}{d_{1}}\right) x_{k} \\
& =\left(\sum_{k}\left(\frac{1}{\sqrt{\sum_{m=1}^{v}\left({ }^{v} D_{i m}\right)}}\right)\left(\frac{1}{\sqrt{\sum_{s=1}^{v}\left({ }^{v} D_{j s}\right)}}\right) \frac{d_{k}}{d_{1}}\right) x_{k}
\end{aligned}
$$

Using the Cauchy-Schwarz inequality and calculating the distance matrices of ${ }^{\delta} X$, we obtain

$$
\lambda_{1}(G) x_{i} \geq\left(\frac{1}{n \sqrt{n}} \frac{1}{n \sqrt{n}}\right)\left(\sum_{k}\left(\frac{d_{k}}{d_{1}}\right)\right) x_{k} .
$$

We know that $\sum_{k=1}^{n}=2 m$. Hence,

$$
\lambda_{1}(G) \geq \frac{2 m}{d_{1} n^{3}}
$$

Secondly, we will show that $\lambda_{2}(G) \leq \sqrt{\frac{2 m\left(\sqrt{2 m n}-d_{1}\right)}{n^{3} d_{1}}-\frac{4 m^{2}}{n^{6} d_{1}^{2}}+m}$.

We know that $\sum_{i=1}^{n} \lambda_{i}=0$ and $\sum_{i=1}^{n}\left(\lambda_{i}\right)^{2}=2 m$. So, $\lambda_{1}+\lambda_{2}=-\sum_{i=3}^{n} \lambda_{i}$. Hence,

$$
\lambda_{2} \leq\left|\lambda_{1}\right|+\left|\sum_{i=3}^{n} \lambda_{i}\right| .
$$

If we take the square of both sides, we obtain

$$
\left(\lambda_{2}\right)^{2} \leq\left(\lambda_{1}\right)^{2}+2\left|\lambda_{1}\right|\left|\sum_{i=3}^{n} \lambda_{i}\right|+\left|\sum_{i=3}^{n} \lambda_{i}\right|^{2} .
$$

By the Cauchy-Schwarz inequality with the above result, we have

$$
\left(\lambda_{2}\right)^{2} \leq\left(\lambda_{1}\right)^{2}+2\left|\lambda_{1}\right| \sum_{i=3}^{n}\left|\lambda_{i}\right|+\sum_{i=3}^{n}\left(\lambda_{i}\right)^{2}
$$




$$
\leq\left(\lambda_{1}\right)^{2}+2\left|\lambda_{1}\right|\left(E(G)-\left|\lambda_{1}\right|-\left|\lambda_{2}\right|\right)+2 m-\left(\lambda_{1}\right)^{2}-\left(\lambda_{2}\right)^{2} .
$$

If we make necessary calculations, we have

$$
\left(\lambda_{2}\right)^{2} \leq\left|\lambda_{1}\right|(E(G))-\left|\lambda_{1}\right|^{2}-\left|\lambda_{1}\right|\left|\lambda_{2}\right|+m
$$

Since $\lambda_{1} \geq \lambda_{2}$ and $\lambda_{1} \leq d_{1}$, then $d_{1} \geq \lambda_{1} \geq \lambda_{2}$. So,

$$
\begin{aligned}
\left(\lambda_{2}\right)^{2} & \leq\left|\lambda_{1}\right|(E(G))-\left|\lambda_{1}\right|^{2}-\left|\lambda_{1}\right| d_{1}+m \\
& \leq\left|\lambda_{1}\right|\left(E(G)-d_{1}\right)-\lambda_{1}^{2}+m .
\end{aligned}
$$

Since $E(G) \leq \sqrt{2 m n}$ and $\lambda_{1} \geq \frac{2 m}{n^{3} d_{1}}$, then

$$
\begin{aligned}
\lambda_{2} & \leq \sqrt{\frac{2 m}{n^{3} d_{1}}\left(E(G)-d_{1}\right)+m-\left(\frac{2 m}{n^{3} d_{1}}\right)^{2}} \\
& \leq \sqrt{\frac{2 m\left(\sqrt{2 m n}-d_{1}\right)}{n^{3} d_{1}}-\frac{4 m^{2}}{n^{6} d_{1}^{2}}+m .}
\end{aligned}
$$

\subsection{Upper and lower bounds on incidence energy}

In the sequel of this paper, we expand bounds under the energy of ${ }^{\delta} X(G)$ with $n, D$ and $\operatorname{det}\left({ }^{\delta} X(G)\right)$.

Theorem 3.3 Let $G$ be a regular graph of order $n$ with $m$ edges. Let $I E(G)$ be an incidence energy of ${ }^{\delta} X(G)$ and $\sigma_{1}, \sigma_{2}, \ldots, \sigma_{n}$ be singular values of ${ }^{\delta} X(G)$. Then

$$
I E\left({ }^{\delta} X_{1}\right)(G)+I E\left({ }^{\delta} X_{2}\right)(G) \leq 2 \sqrt{\Delta}+\sqrt{(n-1)\left(2 \sqrt{2 m n}-\frac{4 m}{n}\right)+2\left|\sqrt{2 m n}-\frac{2 m}{n}\right|} .
$$

Proof Let $\sigma_{i}$ and $\sigma_{j}$ be singular values of $\left({ }^{\delta} X_{1}\right)(G)$ and $\left({ }^{\delta} X_{2}\right)(G)$, respectively. We will use that $\sum_{i=2}^{n}\left(\sigma_{i}\right)^{2}=\sum_{i=2}^{n}\left|\lambda_{i}\right|=E(G)-\left|\lambda_{1}\right|$.

By Lemma 2.5,

$$
\sum_{i=1}^{k} \sigma_{i}\left({ }^{\delta} X_{1}+{ }^{\delta} X_{2}\right) \leq \sum_{i=1}^{k} \sigma_{i}\left({ }^{\delta} X_{1}\right)+\sum_{i=1}^{k} \sigma_{i}\left({ }^{\delta} X_{1}\right)
$$

So,

$$
\begin{aligned}
\sum_{i=2, j=2}^{n}\left(\sigma_{i}+\sigma_{j}\right) & \leq \sum_{i=2}^{n} \sigma_{i}^{2}+\sum_{i=2}^{n} \sigma_{j}^{2}+2 \sqrt{\sum_{i=2}^{n} \sigma_{i}^{2} \sum_{i=2}^{n} \sigma_{j}^{2}} \\
& =E\left({ }^{\delta} X_{1}\right)-\lambda_{1}+E\left({ }^{\delta} X_{2}\right)-\lambda_{1}+2 \sqrt{\left(E\left({ }^{\delta} X_{1}\right)-\lambda_{1}\right)\left(E\left({ }^{\delta} X_{2}\right)-\lambda_{1}\right)} .
\end{aligned}
$$

Since $\lambda_{1} \geq \frac{2 m}{n}$ and $E(G) \leq \sqrt{2 m n}$, we get

$$
\sum_{i=2, j=2}^{n}\left(\sigma_{i}+\sigma_{j}\right) \leq 2 \sqrt{2 m n}-\frac{4 m}{n}+2\left|\sqrt{2 m n}-\frac{2 m}{n}\right|
$$


Since $\lambda_{1} \leq \Delta$,

$$
\begin{aligned}
I E\left({ }^{\delta} X_{1}\right)(G)+I E\left({ }^{\delta} X_{2}\right)(G) & =\sigma_{1}+\sigma_{1}+\sum_{i=2, j=2}^{n}\left(\sigma_{i}+\sigma_{j}\right) \\
& =2 \sqrt{\lambda_{1}}+\sqrt{(n-1) \sum_{i=2, j=2}^{n}\left(\sigma_{i}+\sigma_{j}\right)^{2}} .
\end{aligned}
$$

Hence,

$$
I E\left({ }^{\delta} X_{1}\right)(G)+I E\left({ }^{\delta} X_{2}\right)(G) \leq 2 \sqrt{\Delta}+\sqrt{(n-1)\left(2 \sqrt{2 m n}-\frac{4 m}{n}\right)+2\left|\sqrt{2 m n}-\frac{2 m}{n}\right|} .
$$

Theorem 3.4 Let $G$ be a graph with $n$ nodes and $m$ edges. Let the smallest and the largest positive singular values $\sigma_{1}$ and $\sigma_{n}$ of ${ }^{\delta} X$, respectively, and $\operatorname{det}\left({ }^{\delta} X\right)$ be a determinant of the distance-sum-connectivity matrix ${ }^{\delta} X$ of $G$. For $n>1$,

$$
E(G) \leq \frac{n^{2}}{2(n-1)}\left(\frac{1}{\sqrt[4]{D_{i n} D_{j n}}}-\sqrt{\frac{\operatorname{det}\left({ }^{\delta} X\right)}{\prod_{i=2}^{n-1}}}\right)
$$

where $E(G)$ is the energy of ${ }^{\delta} X$.

Proof Suppose $a_{i}=1$ and $b_{i}=\sigma_{i}, 1 \leq i \leq n$. Apply Theorem 2.3 to show that

$$
\sum_{i=1}^{n} 1^{2} \sum_{i=1}^{n} \sigma_{i}^{2}-\left(\sum_{i=1}^{n} \sigma_{i}\right)^{2} \leq \frac{n^{2}}{4}\left(\sigma_{n}-\sigma_{1}\right)^{2}
$$

Thus, it is readily seen that

$$
n E(G) \leq \frac{n^{2}}{4}\left(\sigma_{n}-\sigma_{1}\right)^{2}+\left(\sum_{i=1}^{n} \sigma_{i}\right)^{2}
$$

By the Cauchy-Schwarz inequality, we can express that

$$
\begin{aligned}
n E(G) & \leq \frac{n^{2}}{4}\left(\sigma_{n}-\sigma_{1}\right)^{2}+\sum_{i=1}^{n} \sigma_{i}^{2} \\
& \leq \frac{n^{2}}{4}\left(\sigma_{n}-\sigma_{1}\right)^{2}+E(G) .
\end{aligned}
$$

Then it suffices to check that

$$
\begin{aligned}
E(G) & \leq \frac{n^{2}}{4(n-1)}\left(\sigma_{n}-\sigma_{1}\right)^{2} \\
& \leq \frac{n^{2}}{4(n-1)}\left(\sigma_{n}^{2}-2 \sigma_{n} \sigma_{1}+\sigma_{1}^{2}\right) \\
& \leq \frac{n^{2}}{4(n-1)}\left(\lambda_{n}-2 \sqrt{\lambda_{n}} \sqrt{\lambda_{1}}+\lambda_{1}\right)
\end{aligned}
$$




$$
\leq \frac{n^{2}}{4(n-1)}\left(\lambda_{n}-2 \sqrt{\frac{\operatorname{det}\left({ }^{\delta} X\right)}{\prod_{i=2}^{n-1}}}+\lambda_{1}\right)
$$

Since $\lambda_{1} \geq \lambda_{2} \geq \cdots \geq \lambda_{n}$ and using Theorem 3.1, we obtain

$$
E(G) \leq \frac{n^{2}}{2(n-1)}\left(\frac{1}{4 \sqrt{D_{i n} D_{j n}}}-\sqrt{\frac{\operatorname{det}(\delta X)}{\prod_{i=2}^{n-1}}}\right) .
$$

\subsection{Upper and lower bounds for matching energy}

We determine an upper bound for the matching energy applying the Polya-Szego inequality, and we give some results using Maclaurin's symmetric mean inequality.

Theorem 3.5 Let $G$ be a connected graph and $M E(G)$ be a matching energy of $G$, then

$$
M E(G) \geq \frac{\sqrt{8 m n s_{1} s_{n}}}{\left|s_{1}\right|+\left|s_{n}\right|}
$$

where $\mu_{i}$ is the zero of its matching polynomial.

Proof Let $\mu_{1}, \mu_{2}, \ldots, \mu_{n}$ be the zeros of their matching polynomial. We suppose that $s_{i}=$ $\left|\mu_{i}\right|$, where $s_{1} \leq s_{2} \leq \cdots \leq s_{n}$ and $t_{i}=1,1 \leq i \leq n$. By Theorem 2.4, we obtain

$$
\sum_{i=1}^{n}\left|\mu_{i}\right|^{2} \sum_{i=1}^{n} 1^{2} \leq \frac{1}{4}\left(\sqrt{\frac{\left|\mu_{n}\right|}{\left|\mu_{1}\right|}}+\sqrt{\frac{\left|\mu_{1}\right|}{\left|\mu_{n}\right|}}\right)^{2}\left(\sum_{i=1}^{n}\left|\mu_{i}\right|\right)^{2} .
$$

Since $\sum_{i=1}^{n} \mu_{i}^{2}=2 m$,

$$
n \sum_{i=1}^{n}\left|\mu_{i}\right|^{2} \leq \frac{1}{4}\left(\sqrt{\frac{\left|\mu_{n}\right|+\left|\mu_{1}\right|}{\left|\mu_{1} \mu_{n}\right|}}\right)^{2}\left(\sum_{i=1}^{n}\left|\mu_{i}\right|\right)^{2} .
$$

It is easy to see that

$$
M E(G) \geq \frac{\sqrt{8 m n\left|\mu_{1} \mu_{n}\right|}}{\left|\mu_{1}\right|+\left|\mu_{n}\right|} .
$$

We can assume that the maximum $\left|\mu_{i}\right|$ is $s_{n}$ and the minimum $\left|\mu_{i}\right|$ is $s_{1}$. So the bound can be sharpened, that is,

$$
M E(G) \geq \frac{\sqrt{8 m n s_{1} s_{n}}}{\left|s_{1}\right|+\left|s_{n}\right|} .
$$

Corollary 3.6 Let G be a k-regular graph. Then

$$
M E(G) \geq \frac{2 n k \sqrt{s_{1}}}{\left|s_{1}\right|+k} .
$$


Proof Since $G$ is a $k$-regular graph, we can take $s_{n}=k$ and $2 m=n k$. By Theorem 3.5,

$$
M E(G) \geq \frac{\sqrt{4 n^{2} k^{2} s_{1}}}{\left|s_{1}\right|+k} .
$$

Hence,

$$
M E(G) \geq \frac{2 n k \sqrt{s_{1}}}{\left|s_{1}\right|+k}
$$

Theorem 3.7 Let $G$ be a connected graph with $n$ vertices and $m$ edges. Then

$$
M E(G) \geq n \sqrt{\frac{2 m}{n-1}}
$$

if and only if $\mu_{1}=\mu_{2}=\cdots=\mu_{n}$.

Proof Let us consider $s=n$ and $x_{i}=\left|\mu_{i}\right|$ for $i=1,2, \ldots, n$. Setting that in Lemma 2.6, we get

$$
M_{1}=\frac{\sum_{i=1}^{n}\left|\mu_{i}\right|}{n}=\frac{M E(G)}{n} .
$$

Also,

$$
M_{2}=\frac{1}{n(n-1)} \sum_{i=1}^{n} \sum_{j=1, j \neq i}^{n}\left|\mu_{i}\right|\left|\mu_{j}\right| .
$$

Since $\sum_{i=1}^{n} \mu_{i}^{2}=2 m$ and $\sum_{i<j}\left|\mu_{i}\right|\left|\mu_{j}\right|=-m$, then

$$
M_{2}=\frac{1}{n(n-1)} \sum_{i=1}^{n} m^{2}=\frac{m^{2}}{n(n-1)} .
$$

We know that $M_{1} \geq M_{2}^{\frac{1}{2}}$. So,

$$
M E(G) \geq \frac{m n}{n-1}
$$

The above equality holds if and only if $\mu_{1}=\mu_{2}=\cdots=\mu_{n}$.

Theorem 3.8 Let $G$ be a connected graph with $n$ vertices and $m$ edges. Then

$$
M E(G) \leq^{2 \ell} \sqrt{(2 m)^{\ell}-n(n-1) \prod_{i=1}^{n}\left|\mu_{i}\right|^{\frac{2 \ell}{n}}}
$$

if and only if $\mu_{1}=\mu_{2}=\cdots=\mu_{n}$. 
Proof Let us consider $s=n$ and $x_{i}=\left|\mu_{i}\right|^{\ell}$ for $i=1,2, \ldots, n$. By Lemma 2.6, we determine

$$
\begin{aligned}
M_{2} & =\frac{1}{n(n-1)} \sum_{i=1}^{n} \sum_{j=1, j \neq i}^{n}\left|\mu_{i}\right|^{\ell}\left|\mu_{j}\right|^{\ell} \\
& =\frac{1}{n(n-1)}\left(\left(\sum_{i=1}^{n}\left|\mu_{i}\right|^{\ell} \mid\right)^{2}-\sum_{i=1}^{n}\left(\left|\mu_{j}\right|^{\ell}\right)^{2}\right) .
\end{aligned}
$$

Using the Cauchy-Schwarz inequality, we have

$$
M_{2} \leq \frac{1}{n(n-1)}\left(\left(\sum_{i=1}^{n}\left|\mu_{i}\right|^{\ell}\right)^{2}-\left(\sum_{i=1}^{n}\left|\mu_{j}\right|\right)^{2 \ell}\right) .
$$

It is clear that the above equality gives

$$
M_{2}=\frac{1}{n(n-1)}\left((2 m)^{\ell}-(M E(G))^{2 \ell}\right) .
$$

Thus, it is pointed out that $(M E(G))^{2 \ell} \leq(2 m)^{\ell}-n(n-1) M_{2}$. Since $M_{2}^{\frac{1}{2}} \geq M_{n}^{\frac{1}{n}}$ and $M_{n}=$ $\prod_{i=1}^{n}\left|\mu_{i}\right|^{\ell}$, then

$$
(M E(G))^{2 \ell} \leq(2 m)^{\ell}-n(n-1)\left(\prod_{i=1}^{n}\left|\mu_{i}\right|^{\ell}\right)^{\frac{2}{n}}
$$

Hence,

$$
M E(G) \leq \sqrt{(2 m)^{\ell}-n(n-1) \prod_{i=1}^{n}\left|\mu_{i}\right|^{\frac{2 \ell}{n}}}
$$

The above result holds if and only if $\mu_{1}=\mu_{2}=\cdots=\mu_{n}$.

\section{Conclusions}

The main goal of this work is to examine distance-sum-connectivity matrix ${ }^{\delta} X$. We find some upper bounds for the distance-sum-connectivity matrix of a graph involving its degrees, its edges, and its eigenvalues. We also give some results for the distance-sumconnectivity matrix of a graph in terms of its energy, its incidence energy, and its matching energy.

\section{Acknowledgements}

The author would like thank for the valuable suggestions of referees.

\section{Funding}

Not applicable.

Competing interests

The author reports that she has no competing interests. 


\section{Publisher's Note}

Springer Nature remains neutral with regard to jurisdictional claims in published maps and institutional affiliations.

Received: 22 May 2018 Accepted: 30 June 2018 Published online: 13 July 2018

\section{References}

1. Bapat, R.B.: Graphs and Matrices. Indian Statistical Institute, New Delhi (2010)

2. Biler, P., Witkowski, A.: Problems in Mathematical Analysis. Marcel Dekker, New York (1990)

3. Cretkovic, D., Doob, M., Sachs, H.: Spectra of Graphs-Theory and Application. Academic Press, New York (1980)

4. Das, K.C., Kumar, P.: Some new bounds on the spectral radius of graphs. Discrete Math. 281, 149-161 (2004)

5. Fan, K.: On a theorem Weyl concerning eigenvalues of linear transformation I. Proc. Natl. Acad. Sci. USA 35, 652-655 (1949)

6. Farrell, E.J.: An introduction to matching polynomials. J. Comb. Theory, Ser. B 27, 75-86 (1979)

7. Gutman, I.: The energy of a graph: old and new results. In: Betten, A., Kohnert, A., Laue, R., Wassermann, A. (eds.) Algebraic Combinatorics and Applications, pp. 196-211. Springer, Berlin (2001)

8. Gutman, I., Kiani, D., Mirzakhah, M.: On incidence energy of graphs. MATCH Commun. Math. Comput. Chem. 62 573-580 (2009)

9. Gutman, I., Kiani, D., Mirzakhah, M., Zhou, B.: On incidence energy of a graph. Linear Algebra Appl. 431, 1223-1233 (2009)

10. Gutman, I., Li, X., Zhang, J.: Graph energy. In: Dehmer, M., Emmert Streib, F. (eds.) Analysis of Complex Networks. From Biology to Linguistics, pp. 145-174. Wiley-VCH, Weinheim (2009)

11. Gutman, l., Matelijević, M.: Note on the Coulson integral formula. J. Math. Chem. 39, 259-266 (2006)

12. Horn, R.A., Johnson, C.R.: Matrix Analysis. Cambridge University Press, New York (1985)

13. Joojandeh, M.R., Kiani, D., Mirzakhah, M.: Incidence energy of a graph. MATCH Commun. Math. Comput. Chem. 62 , 561-572 (2009)

14. Li, X., Shi, Y., Gutman, I.: Graph Energy. Springer, New York (2012)

15. McClelland, B.J.: Properties of the latent roots of a matrix: the estimation of $\pi$ electron energies. J. Chem. Phys. 54, 640-643 (1971)

16. Ozeki, N.: On the estimation of inequalities by maximum and minimum values. J. Coll. Arts Sci., Chiba Univ. 5, 199-203 (1968)

17. Polya, G., Szego, G.: Problems and Theorems in Analysis. Series. Integral Calculus. Theory of Functions. Springer, Berlin (1972)

18. Vasudev, C.: Graph Theory with Applications. (1). New Age International Publishers, New Delhi (2006)

\section{Submit your manuscript to a SpringerOpen ${ }^{\circ}$ journal and benefit from:}

- Convenient online submission

- Rigorous peer review

- Open access: articles freely available online

- High visibility within the field

- Retaining the copyright to your article

Submit your next manuscript at $\boldsymbol{\text { springeropen.com }}$ 